\title{
Reports and Surveys
}

\section{ADVANCES IN BIOROBOTIC VISION}

The Australian National University Biorobotic Vision Group has described its innovative researches and developments in the field of robotic vision. The group has been researching the principles by which insects see, control flights and navigate, in order to transfer their findings to their work in robotic vision. They believe this work will help them in their aim to devise algorithms for machine vision and autonomous, visually guided robots. In particular, this work is in reference to using optic flow to measure image motion.

The researchers have already successfully developed a hover controller that can hover over an arbitary set of landmarks with no manual intervention.They have also designed an autonomous helicopter that will hover and fly without using GPS.

\section{Motion-sensitive visual systems}

Both these prototypes use a motion-sensitive visual system that determines the apparent angular rate of the ground plane in the longitudinal and lateral directions. Height is determined from the speed of the craft and measuring of optical flow. The autonomous helicopter is said to have successfully flown for a distance of $2 \mathrm{~km}$ without the use of GPS and was guided by 'looking around' for itself using this innovative system.

\section{Panoramic imaging system}

Research on panoramic imaging systems which use a standard video camera viewing a specially shaped reflective surface, is also in hand. This system is being developed for use in surveillance systems and for visual guidance and the control of autonomous. A number of projects to produce such devices are being carried out throughout the world at a time when 'drone systems' are deemed to be preferable to human controlled ones. A great deal of work in this direction is being carried out at defence establishments.

\section{Further robot vision projects}

The Biorobotic Group are also developing a number of other systems. They include:

- Corridor following robot

- A terrain-following autopilot for an autonomous helicopter

- A robot that measures visual image deformation to estimate its own motion in the environment

- An obstacle-avoiding robot.

These cannot be achieved without progress by the biorobotics researchers into the construction of algorithms for using image deformation to estimate self-motion in two and three dimensions and for the estimation of self-motion from optical flow. There is enormous scope for the uses of these types of systems over a whole range of applications and in particular, the development of intelligent 'drone' aircraft in both military and other applications. The need for robots that are more 'intelligent' remains and their use in applications that range from domestic and industrial to defence and civil disasters is ever present.

\section{BIOLOGICALLY-INSPIRED ROBOTICS}

\section{Pioneer neurophysiologist}

Robotics researchers will already know of the pioneering work of the neurophysiologist William Grey Walter. He was a world leader in many endeavours and, in particular, in electroencephalography. His work and contribution to science was recalled last year at a workshop held at the Hewlett-Packard Laboratories at Bristol (UK), when Dr Owen Holland (University of Essex, UK) gave an insight into Dr Grey Walter's life and work. This was a workshop that set out to highlight research in the field of biologicallyinspired robots. The attendance at this event indicated the interest in this research area and many who attended were surprised at the high number of postgraduate students and international delegates who were present.

We were told that Dr Grey Walter was among the first to grasp the potential offered by mobile autonomous robots to test how high-level observable behaviours might arise from low-level sensory, neural and motor processes. This potential Dr Holland said:

"was embodied in the world-famous 'tortoise' mobile robots. In Grey Walter's own words 'these machines are perhaps the simplest that can be said to resemble animals. Crude though they are, they give an eerie impression of purposefulness, independence, and spontaneity"”.

\section{Current and future developments}

This research gathering was sponsored by the UK's Engineering and Physical Sciences Research Council (EPSRC) and the BBSRC in support of the Adaptive and interactive Behaviour of Animal and Computational Systems (Aibacs). This is another initiative of the EPSRC at the interface of biology, engineering and computing. Some of the internationally acclaimed practitioners across a range of the relevant disciplines put the subject in perspective. For example, Rodney Brooks of MIT's AI Laboratory (US), gave a critical view of the past, present and future of the bioinspired robotics. He believes that:

"the field needs some application successes, tools to understand biological systems, and the consideration of features of biological creatures such as metabolism".

Further comments on future prospects were made by Michael Arbib of the University of Southern California (US), who said that: 
"There is a lot of biology out there waiting to insplre researchers in biologically-inspired robotics".

It was interesting to note that whilst the field is in dire need of some inspired strategies for biologically-motivated research, many scientists still refuse to take an interdisciplinary view of robotics.

Contributions, however, are invited from any readers with thoughts on the subject either to Robotica, or by contacting directly those involved with this reported workshop (Mark.hylton@epsrc.ac.uk.)

It should be noted that the proceedings of this particular workshop have been published as a web-accessible HewlettPackard Technical Report. The link with the arts is further highlighted by the publication of the selected papers from this reported event as a special issue of the: Philosophical Transactions of the Royal Society (Part A).

\section{BUSINESS \& ENTERPRISE}

\section{UK automation \& control systems}

A recent analysis* of UK Automation \& Control Systems industry reveals that $88 \%$ of the companies involved is using formal debt to run their company. We are informed that for $14 \%$ of the market the level of debt is dangerously high. The report poses the question "How many of these companies would admit to running their business through debt?".

The analysis indicates that:

"Borrowing is endemic. Only 27 companies in the industry do not carry any debt. The average company in the UK Automation \& Control Systems industry is financing around $30 \%$ of their assets. Based on an average margin of $2.6 \%$, paying back this debt is a very unlikely scenario given $32 \%$ of the industry is currently making a 1oss".

The analysis also asks the question and comments:

"Who is responsible at the 32 companies where the level of debt has risen to such a high level that even normal business trading has become hazardous? These companies are struggling. Their debts have increased nearly $68 \%$ in the last 3 years and their ability to pay these debts back is under great threat

These 32 companies are using debt as a permanent method of financing their company. All have carried some sort of debt for the last 4 years".

A further analysis by the compilers reveals, we are informed, that of these 32 companies:

- 24 are loss making. This extra debt is financing losses in an already leaking ship.

- 20 failed to increase sales. This extra finance is not delivering market growth.

\footnotetext{
* Editor's note: This report was received in March 2003. An update and a copy of the Analysis can be obtained from: Plimsoll Portfolio Analysis, 1st Edition 2003, which contains an individual analysis, the publishers say, of the top 465 UK Automation \& Control Systems. See also: www.plimsoll.co.uk
}

- Interest payments are eating almost $1.4 \%$ of sales for all 32 companies. Staying competitive will certainly become a problem.

- All 32 are placed in "Danger" by the analysts own financial rating system. The implications are severe.

It concludes by forecasting that:

"An exit route for many of these 32 companies will come through a buy out from a stronger predator exploiting their adversity. Six of these are named in the analysis as acquisition prospects good companies smothered by debt.

The analysis reveals those that are already heavily in debt and will serve as an early warning to those that are considering dancing with the devil".

\section{Survey of UK business interaction}

A research report published in the Higher Education Business Interaction Survey shows that British universities are establishing stronger commercial links with the business sector. The report says that the universities:

"are involved in more spin-off companies and have an increasing role in economic development. By comparison with North America, UK universities generated one spinoff firm for every $£ 8.6$ million of research expenditure, while in Canada the ratio was one for every $£ 13.9$ million and in the USA, one for every $£ 53.1$ million”.

\section{Link Information Storage \& Display System}

The UK government announced a new $£ 24$ million programme to support the UK's computer and information technology industry. A new LINK information Storage and Display programme has been set up to fund a range of collaborative projects between companies and the universities, with the aim of helping UK-based companies to exploit the global market which could reach more than $£ 100$ billion by the end of the decade.

Half of the funding is provided by the Department of Trade and Industry and the Engineering and Physical Sciences Research Council.

\section{UK Manufacturing Centres}

Innovative manufacturing centres have been set up in the U.K., and a government report has announced that:

"12 university-based Innovative Manufacturing Centres have been created as part of a Government-backed $£ 60$ million manufacturing initiative. Located at Cambridge, Loughborough, Salford, Liverpool, Reading, Warwick, Nottingham, Bath, UCL and Cranfield, the centres will be led by a team of academics and research specialists and will support all aspects of manufacturing from aerospace to bio-pharmaceuticals. The centres will be working in partnership with industry, with a strong emphasis on business processes research to ensure that activities add value and maintain competitive edge in the market place". 


\section{LANGUAGES FQR ROBOTS}

A recent report by Dr Tim Oates, a robotics expert from the University of Maryland-Baltimore, USA, summarises the advances being made by scientists in developing language systems for talking to robots. We are all aware that it is now becoming possible to have a dialogue with a computer system so that, in consequence, we should be able to communicate using a language with a suitably computerised machine. The language lessons given to computer systems by artificial intelligence experts and computer scientists could equally be given to robots so that they too can respond reasonably intelligently using speech. These are the skills we need to embed in our robots if they are, indeed, to reach their ultimate potential as humanoid helpers, whether in the industrial areas or in domestic applications. We also know that over the last decade robots have often been claimed to be 'intelligent'. Certainly, they have advanced beyond our earlier predictions. They can now, of course, walk on their own legs, climb stairs, traverse unknown environments and carry out numerous other activities, many of which have been recorded in this section.

Robotic systems are still, however, restricted in their abilities in both movement, and particularly in their response to humans and, indeed, their fellow robots. Designing robots that can respond has proved extremely difficult and progress has been slow. It is, of course, not too difficult to build systems that respond to stored commands when these commands are identified. Carrying out a dialogue, as Turing soon realised, is very much another matter. Dr Oates puts it very simply when he writes:

"Teaching them is very hard. Imagine you are in a foreign country and don't know the language. You can't even tell where a word begins and ends, much less the meaning of the word".

Aware of these serious difficulties, Dr Oates has set his goal as being able to make a robot follow him around the university campus, gathering and processing information. He has hopes that in say a year from now, he will be able to tell the robot to 'look out for that trash can' or 'let's go through that door'. In five years time he aims to be able to say: 'Could you go into the room we were just in and bring me the red ball'. He considers this to be a very ambitious but not an unreasonable goal. Many of the problems Dr. Oates will have to solve are those already being tackled by researchers in artificial intelligence $(A I)$. There must obviously be a transfer of research information from the human-machine dialogue studies to robotic systems. We are told by Dr. Luc Steels an AI researcher with Sony Computer Science Laboratory in Paris that it is:

"now becoming possible to have open-ended dialogues with physically embedded robots".

Researchers are now experimenting with a variety of techniques to enable robots to use language. In some experiments, we are told, robots are even at the stage where instead of copying their human instructors they have invented their own words and grammatical structures in the same way as human-computer conversations have been developed. In both techniques the methods used resemble the way a parent or older sibling teaches a child to associate a spoken word with an object. Dr Steels says that in an experiment the method used resembles:

"the way a parent teaches a child, the 'pupil' was an enhanced version of AIBO, a popular robotic toy dog marketed by Sony, the Japanese electronics company, which is supporting this work.

The little robot was equipped with eyes (a video camera), ears (a microphone), a voice box and a computer program able to recognize and pronounce human words. We pre-programmed AIBO to recognize a few spoken words like 'look', 'listen', 'what is it?', 'good',.

He has published an account of this study on the Internet where he has described a typical learning session involving AIBO. To accomplish a given task the robot had to connect two very different electronic patterns, one from the sound waves coming to 'his ears' and the other coming from 'his eyes'. Dr Steels says that:

"We have carried out a realistic robotic experiment in the sense that the robot is not only ignorant about the words in the language, but also about the concepts underlying these words".

Dr Tim Oates has also conducted experiments where there is no direct instruction by a human teacher. Instead, the robot must learn the language on its own by observing what people do and say In one experiment he arranged a set of coloured blocks in various patterns, while volunteers described what he was doing in simple sentences. The robot watched and listened. He reports that after hearing fifty statements describing the same scene over and over again, the robot was able to associate certain sound patterns with specific objects such as 'blue block', store them in its computer memory, and repeat them when asked. He writes that:

"The system successfully discovered words and their denotations in three different languages: English, German, and Mandarin Chinese".

Further experiments have been carried out by researchers to study an approach where two robots teach each other languages with virtually no human intervention. In this example of 'unsupervised learning' it was shown that gradually the two robots built up a common vocabulary of about 100 words which, although basic, expressed such concepts as up, down, left, right, red, green, large and small.

To many AI specialists this mimics their own experiments of computers that learn from one another. In these cases confirmation is accorded to the belief that robots/computers can learn the meaning of words in a number of different ways.

It is, of course, to be hoped that all scientists working on this form of interactive communication will interact themselves and share their endeavours and findings for the sake of the common purpose of advancing technology. 


\section{PRODUCT LAUNCHES}

\section{Nev robot for cleaning}

ABB offers the Tricept robot which,they claim.provides cost-efficient flexibility for processing cast automotive parts. The company say that:

"ABB, the global power and automation technologies group, has launched the IRB 940 Tricept robot to complement its extensive range of foundry automation equipment. The IRB 940 is an exceptionally powerful and robust robot developed for the robotic cleaning and premachining of aluminium castings for the automotive industry".

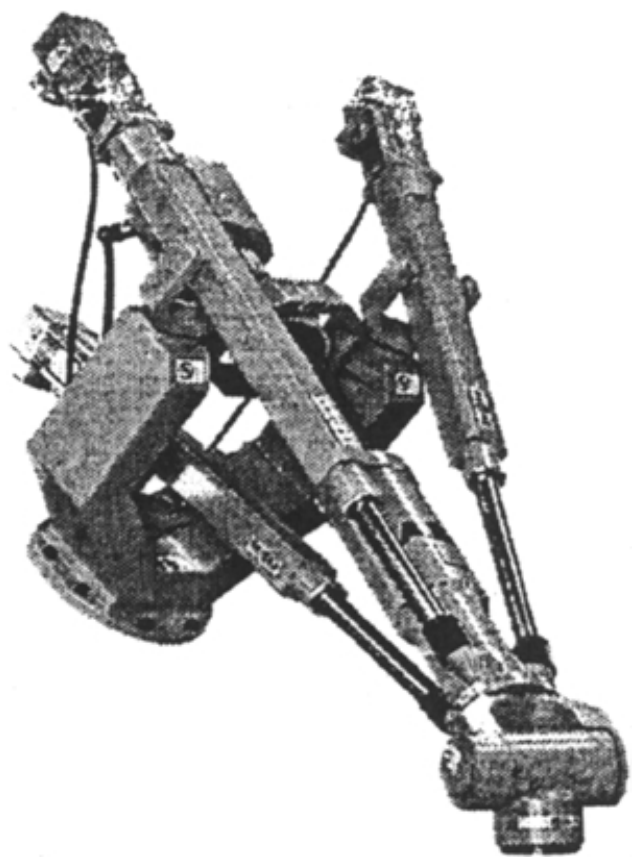

Fig. 1. IRB 940 Tricept.

ABB has supplied over 100,000 robots worldwide and has a leadership commitment to robot-based foundry automation which ranges from the smelter to casting and cleaning of parts. The company believe that over the last 30 years, more than 10,000 of their robots have provided improved parts quality, higher productivity and better working conditions in foundries across the world. In their description of their product $\mathrm{ABB}$ write that:

"The IRB 940 Tricept, the product of an alliance between ABB and Sweden-based machine builder SMT Tricept, offers superior performance for the demanding application of heavy cleaning and pre-machining of aluminium castings. The robot is a combination of a mechanical arm built by SMT Tricept and ABB's well-proven S4Cplus robot controller. Having the same controller for both conventional arm-type robots and the Tricept robot on the same production line provides benefits for the end user customer in terms of a common programming language, ease-of-use, spare parts and training. All arm robot functions in the S4Cplus controller are supported for IRB 940 Tricept. Based on ABB's proven leadership in motion control with the S4Cplus the IRB 940 Tricept has excellent motion performance with an acceleration of approximately $1 \mathrm{~g}$, an absolute accuracy of approximately $0.008^{\prime \prime}(0.2 \mathrm{~mm})$, and repeatability of approximately $(0.02 \mathrm{~mm})$.

In comparison with conventional arm robots, the IRB 940 Tricept is an extremely robust structure offering a vertical machining power of $1300 \mathrm{~kg}$ and a horizontal machining power of $350 \mathrm{~kg}$. This robot has the ability to perform machining applications that normally can only be done by machine tools, but still keeping the flexibility and lower cost of an industrial robot. The Tricept design is based on a parallel kinematics framework, best illustrated as an up-side down camera stand, featuring three linear actuators with a center tube in the middle and three additional axes on the wrist".

$\mathrm{ABB}^{*}$ plans to offer the robot to a limited number of system integrators with extensive aluminium part cleaning experience. The robot is part of an ABB program aimed at developing an improved robot-based flexible automation product for aluminium parts manufacture.

\section{TM Robotics}

TM Robotics $\dagger$, which is Toshiba Machine's European industrial robots partner, has publicised its new SR-120HSP SCARA robot (Fig. 2). The company claim that their product has significant control and productivity benefits and the largest work envelope of a robot of its kind. They say that:

"The robot's SR7000 controller uses absolute encoder feedback, rather than incremental signals. This provides high accuracy and dramatically decreases cycle times by removing the need to implement zero return on start up following a shut down.

The 32-bit controller integrates easily with other industrial automation equipment and is capable of simultaneously processing input and output signals during robot motion. Therefore, no time is wasted while signal processing is completed.

The controller is capable of controlling up to 5-axes simultaneously, with options for PTP, short cut, linear interpolation and circular interpolation motion modes. With a total memory capacity of 6,400 points $(12,800$ steps), the controller also offers a multitask function, allowing a maximum of four tasks.

The SR-1204HSP is also extremely accurate, offering repeatability to within $+0.03 \mathrm{~mm}$. Utilising state-ofthe-art technology, the SR-HSP series uses parallel processing to offer smooth, fast motion combined with an extremely sophisticated set of control instructions to suit all applications.

The SR-1204HSP has an arm length of $1200 \mathrm{~mm}$, which means the SCARA can offer nine types of arm configuration ranging from $250 \mathrm{~mm}$ to $1200 \mathrm{~mm}$, making the range one of the best in the industry. This latest model also offers a vertical motion of either $200 \mathrm{~mm}$ or $400 \mathrm{~mm}$

\footnotetext{
* ABB can be accessed on www.abb.com

$\dagger$ Further information from www.houston-associates.com
} 


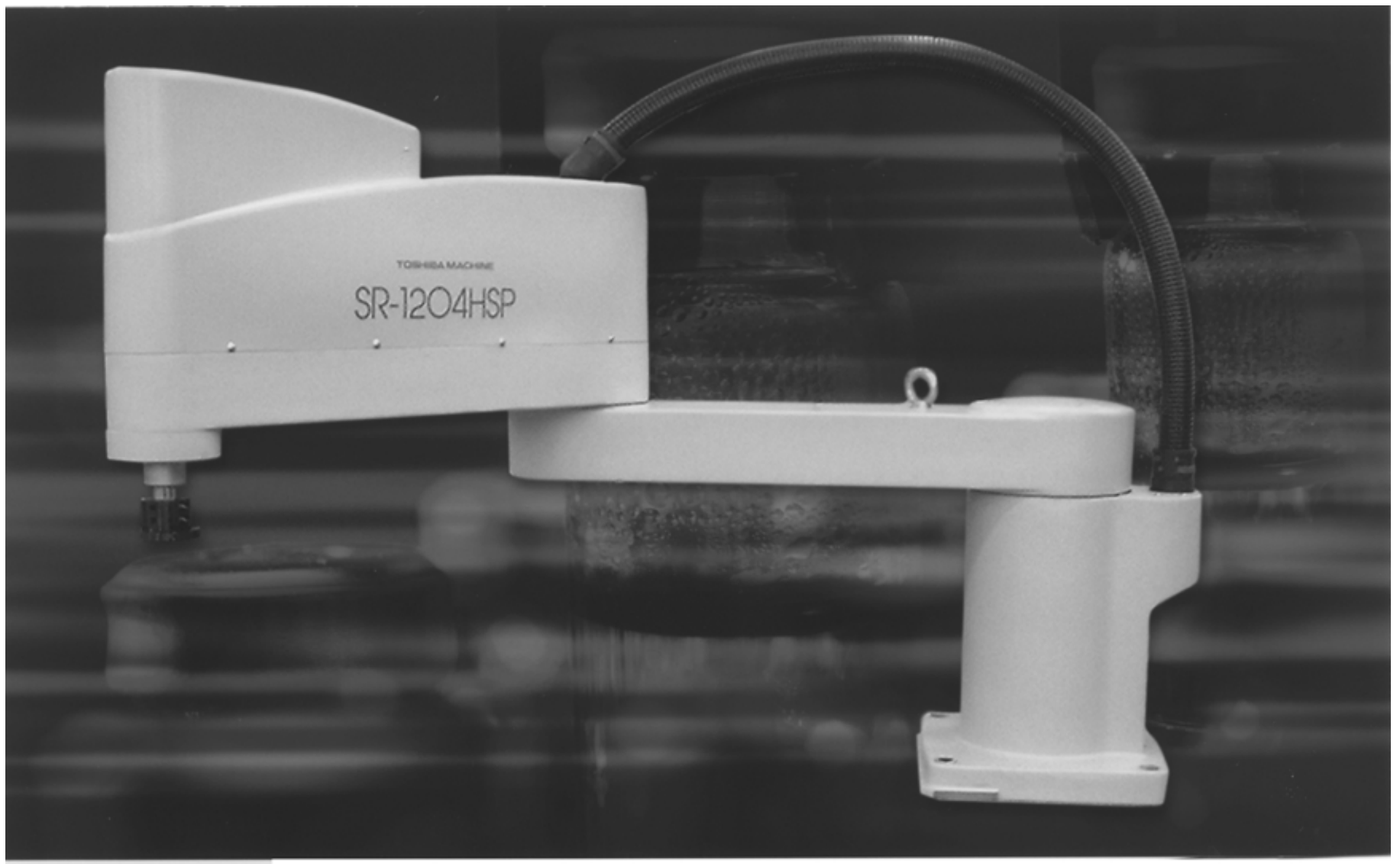

\section{T0SHIBA} MACHINE

Fig. 2. Robotics SR-12040 SCARA robot.

and the robot's reinforced arm can handle up to $15 \mathrm{~kg}$ payload".

\section{REPORTS WORLDWIDE}

\section{Computer modelling}

Researchers at the University of Salford (UK) are expanding the concept of three-dimensional modelling to an unlimited number of dimensions. The $3 \mathrm{D}$ to $\mathrm{nD}$ project combines:

- computer modelling

- simulation techniques

- visualisation

with the use of virtual reality to enable building designs, not only to be seen, but also, to be actively tried. This is achieved by systems that allow the users to virtually walk through and also around the designs displayed. In addition, we are told that the design tool that has been developed is called the ' $\mathrm{nD}$ tool' and will portray not only the design of the building, but also its future.

Earlier this year an international workshop was held at the Mottram Hall, Manchester (UK) to discuss the developments in $3 \mathrm{D}$ to $\mathrm{nD}$ modelling. It is reported that over 50 experts from world-leading industrial and academic organisations in the field took part. This workshop, which was co-chaired by Stanford University (USA) discussed a number of relevant topics and, in particular, multi-design perspective product modelling. It considered that $\mathrm{nD}$ modelling is an interactive process that allows the flexible choice of accessibility, maintenance, sustainability, energy, acoustics, and other design features which should be viewed against construction costs and project schedules. In addition, the discussions examined some of the barriers and enablers for an industry-wide implementation of $\mathrm{nD}$ modelling, and the strategies for a way forward were identified.

The Director of the Research Institute for the Built and Human Environment at Salford University, Professor Ghossan Aouad, summed up the discussions of this high-profile meeting:

"The outcome of the workshop will not only have a profound effect on construction projects of the future, but will also provide the opportunity to combine research efforts and collaborate new ideas with other organisations from across the world".

A report of the workshop proceedings is being published. The project is in receipt of funding from the UK's Engineering, and Physical Sciences Research Council (EPSRC) at the level of $£ 0.5$ million over four years.

For further information contact: g.aouad@salford.ac.uk, and see also: http://ndmodelling.scpm.salford.ac.uk 
2. Using the human body as an electronic circuit

An innovative project has been described by the Nippon Telegraph and Telephone Corporation in Japan, to harness the human body's capacity to conduct electrical signals. The technology being currently researched aims to use the human body to improve communication. The body would be used to function at broadband speeds and allow signals to circulate through it.

As a result, no wires would be needed for the transmission of any signals that may be required for communication between humans. A scenario is presented where a mere handshake could be enough to pass information such as identification, exchange of telephone numbers, email addresses or other messages. Other suggestions for applying the body's electrical conducting properties include entrance to security areas, access to computer systems, as well as many more activities where data has to be exchanged. Such systems could well be used in conjunction with embedded electronic devices which are currently being tested worldwide.

\section{Bacteria used as a database}

A solution to the problems of storing data was suggested in the New Scientist (January, 2003). Data could be encoded as artificial DNA and stored within the genomes of multiplying bacteria and then, it claims, accurately retrieved.

The main concern of the scientists developing such systems is that nearly all of the current ways of storing data, such as paper or electronic, allows it to be lost or destroyed. The users of some electronic systems are well aware that valuable business, industrial data can be corrupted and files destroyed, often at the press of the wrong button. Hence the perceived need to create new types of memory systems.

Research in the US has already shown that data stored as artificial DNA can be stored and processed. Dr Pak Chung Wong of the Pacific Northwest National Laboratory, Washington State (US), is an information technologist who has expressed concern about the protection of valuable information in the case of a nuclear catastrophe. We are told that bacteria may be an inexpensive and stable long-term means of data storage. The laboratory conducting this research was set up as a nuclear energy research institute so that it has a particular need to find alternative and more secure data storage. A report of the laboratories work describes the researchers approach and experimentation. The scientists, Dr Wong says:

"took the words of the song It's a Small World and translated it into a code based on the four 'letters' of DNA. They then created artificial DNA strands recording different parts of the song".

These DNA messages, each about 150 bases long, were inserted into bacteria such as $\mathrm{E}$ coli and Deinococcus radiodurans.

The latter is especially good at surviving extreme conditions. It can tolerate high temperatures, desiccation, ultraviolet light and ionising radiation doses 1,000 times higher than would be fatal to humans.

The beginning and end of each inserted message have special DNA tags devised by the scientists. These "sentinels" stop the bacteria from identifying the message as an invading virus and destroying it.

The magic of the sentinel is that it protects the information, so that even after a hundred bacterial generations we were able to retrieve the exact message".

The researchers tell us that once the DNA message is in the bacteria it is well protected and can survive. It has been estimated that just one millilitre of liquid can store as many as billion bacteria.

If this innovative system of storage proves viable then the potential for the storage of data in a secure environment is enormous. New secure storage systems based on bacteria will then be able to offer an unlimited capacity for protected data.

\section{Supergen programme}

A UK initiative called Supergen* for sustainable power generation and supply was launched in the Spring of 2003

Supergen will invest over $£ 25 \mathrm{~m}$ during a five-year cycle to address the broad challenges of sustainable power generation and supply.

A report 'Powering the Future' issued by the UK Engineering and Physical Sciences Research Council (EPSRC) says that:

"There is a general recognition that the first few decades of the 21 st century will witness a significant change in focus of energy supply in the UK. The main drivers for this include political, social, environmental, and economic factors covering climate change, fossil fuel extraction rates, emissions control, and public awareness of environmental concerns".

The UK's Royal Commission on Environmental Pollution report 'Energy - The Changing Climate' highlighted the need to consider a sustainable approach to power generation in terms of low- or zero carbon technologies This is a view that is supported in Britain by a number of recent studies, including the government's Foresight initiative and the work of the UK's Cabinet Office Performance and Innovation Unit Energy Review.

The first awards given under this initiative have supported:

- Marine Energy: energy from the seas around the coastline

- Hydrogen: the fuel of the future?

- Biomass: that is using fast growing crops as a renewable fuel supply

- Networks: to ensure a reliable supply of power to the UK.

To help this endeavour, EPSRC is already supporting consortia of universities, industry users, and stakeholders to tackle what are seen as strategically important areas. It is also working with other UK Research Councils in its initiative. Obviously this is an initiative which should not be confined to one country and it is one which reflects the challenges that other nations worldwide are also tackling.

* Further details of the Supergen initiative can be obtained from: EPSRC's Edward Clarke - email: edward.clark@epsrc.ac.uk 


\section{Materials research}

We are told that as an alternative to steel, zirconia suffers from one major weakness and that a current UK-funded reseach project is changing that. The overall leader of the research is Professor Mark Rainforth of Sheffield University's Department of Engineering Materials, UK. The research has received two grants from the UK's funding body for engineering, and the physical sciences, * with the total amount of support reaching $£ 530,000$. Describing the progress of the work, researchers write that:

"Improved cutting tools, stronger hip joints, and better medical instruments should result from research looking at ceramic alternatives to steel.

Zirconia, a ceramic with steel-like strength and hardness and high resistance to wear and chemical corrosion, is potentially well suited to industrial, medical and other uses. It produces blades which are much sharper and smoother than steel. and which last up to 50 times longer.

To date, the use of zirconia has been limited by its loss of strength and its subsequent cracking when subjected to temperatures of $100-600^{\circ} \mathrm{C}$ in the presence of water - a process known as hydrothermal degradation. The research team has inhibited this process without compromising the toughness of zirconia by adding trace quantities of materials such as alumina".

The research work is carried out as a collaborative effort with the UK Universities of Sheffield, Glasgow, and Leeds, and with Dynamic Ceramic a small UK company. The scientific underpinning for the company is provided by Sheffield University which has allowed it to develop a production route for a zirconia that is resistant to hydrothermal degradation.

Professor Rainforth says that:

"Increased industrial productivity and improved comfort for hip replacement patents are just two of the many benefits that could result from overcoming zirconia's Achilles heel".

The techniques used in the work include field emission gun TEM and high-energy resolution XPS (at Daresbury, UK). These techniques we are informed, allow the researchers to probe microstructure at the atomic scale, allowing the determination of atom type, position and bonding between atoms.

\section{REVOLUTIONARY COMPUTER SCREEN}

A report published in the journal Nature (May, 2003) provides details of the development of a new computer screen that, it is claimed is 'paper thin'.

The new display is being developed by the $\mathrm{E}$ Ink Corporation, Cambridge, Massachusetts (USA). Dr Yu Chen of the Corporation says that:

"The liquid crystal display is less than $0.3 \mathrm{~mm}(0.01 \mathrm{in}$. thick and can be bent up to $15 \mathrm{~mm}$ (0.6 in.) without distorting the picture or reducing its contrast. It can be

\footnotetext{
* Further details contact: m.rainforth@sheffield.ac.uk
}

viewed from almost any angle, and the screen can even survive being rolled into a cylinder just $4 \mathrm{~mm}$ wide".

Such a computer screen is therefore as thin as a piece of paper and will signify an advance in the development of a true 'e-paper" for electronic books, journals, newspapers, etc. The developers say that, although the current prototype is not a 'true e-paper', since it cannot be bent or folded through any angle without destroying it, it is still an advance in the technology. Eventually, we are told, it may allow the creation of even thinner and more flexible screens for use in wearable computers and electronic newspapers and notepads.

\section{Dr Chen writes that:}

"The technology works using a thin array of transistors connected to millions of tiny capsules of chargesensistive white and black pigment. Negative voltage causes the white particles to move to the surface while positive voltage brings black to the top to create text.

The pixel density is high with 160 pixels on the horizontal axis and 240 on the vertical, as is the resolution at 96 pixels per inch. The display can be updated in a quarter of a second making it fast enough for electronic newspapers, but not for video film.

The use of electronic ink technology on such an ultrathin, and flexible substrate should greatly extend the range of display applications".

The importance of this development and the trend to ' $\mathrm{e}$ paper' cannot be overemphasised. Such new initiatives will continue to influence the way in which we obtain our information and, in particular, such a flexible medium will indeed revolutionise publishing.

Since the initial announcement of this advance Dr Chen and his colleagues at the E Ink Corporation have discussed further developments and applications of their innovative display. They say that:

"Newspapers and books can be displayed by the user at a flick of the switch on a wearable computer screen. It is already possible to create a credit card that when swiped displays a signature and other personal information which will allow banks and retail outlets to check the identity of their customers. Such a flexible display would be suitable to publish a newspaper which could easily be updated by a wireless signal and the system could be available within two years. The display would be updated in a quarter of a second".

The future of academic journals will change from electronic publishing on the internet, for example, to one where their contents will be available on a wearable screen. The electronically printed book, which is available at some retail outlets, will be accessible on the ultra-thin flexible electronic ink screen that promises to revolutionise the way in which we deal with information.

\section{UK RESEARCH PROJECTS}

Innovative proposals with potentially far-reaching consequences are underway at a number of British research establishments. A programme of funding called 'Basic 
Technology Research' has been undertaken by the government's Research Councils, with the aim of creating fundamentally new capabilities for pursuing futuristic research. The idea being that anyone who is able to demonstrate how their research would contribute to a generic technology base that can be adapted to a diverse range of research problems and challenges that spans the interest of all the research councils, should be encouraged to apply for funding. Those who have been awarded funding range from optical biochips to very fast genome sequencing technologies with common threads - of solid-state and nanotechnologies.

Recent funding has been given to the following projects at British Universities (the grant, project leader and university are included for reader reference, in brackets after the project title: for further details of the Basic Technolog Programme see footnote*.

\section{Attosecond technology - light sources, metrology and applications}

\section{“(£3.6m, Tisch, Imperial College London)}

A proper understanding of the way in which the motion of electrons affect the fundamental processes of chemistry, biology and materials science requires probes into attosecond timescales. This project aims to deliver a source of isolated attosecond optical pulses that will provide the scientist with the tools to study these fundamental processes directly for the first time".

\section{Putting the Quantum into information technology}

\section{“(£3.6m, Stoneham, University College London)}

Quantum computing and information technology exploit universal gates. This project centres on a new concept for J-gates, which control the entanglement of two spins. The aim is to make this new class of quantum gates in a system which is silicon-compatible, and to demonstrate the operation of these gates in a representative computation at a useful temperature (liquid nitrogen or above)".

\section{Optical Biochips}

\section{"(£2.1m, Smith, University of Wales, Cardiff)}

This project aims to bring down to a microscale all of the main components used to analyse biological samples in a modern life sciences laboratory, including lasers the size of a human cell, to create an optical lab-on-a-chip. Potential benefits include increasing the success rate of drug discovery, genomics research, disease diagnosis, and operation of ultra-fast computers".

\footnotetext{
* The Basic Technology Programme's Manager is Dr Alasdair Rose: email: alasdair.rose@epsre.ac.uk
}

Four billion bases a day - practical individual genome sequencing

“( $£ 4.8 m$, Bradley, Southampton)

A fundamentally new method of synthesising, screening and sequencing DNA at a rate that is thousands of times faster than existing methods will allow over four billion bases to be sequenced in less than a day on one instrument. If successful, the technique will forever change the landscape of biological and medicinal sciences".

\section{Hyperpolarised technologies for medical and materials science}

\section{“(£1.9m, Morris, Nottingham)}

This project will bring together established UK research communities in magnetic resonance, semiconductors and neutron scattering to develop and enhance technologies for hyperpolarising materials and for storage and transport of these materials. The possibility of devising novel techniques for transferring the polarisation to other materials of interest will be explored."

\section{Cryogenic instrumentation for quantum electronics}

“(£3.1m, Briggs, Oxford)

Many of the most exciting developments in quantum devices need to operate at extremely low temperatures, supported by several stages of electronics operating at successively lower temperatures. This project will develop generic electronics platforms and very low power circuits so that candidate nanoelectronic technologies can be measured and complete circuit designs characterised".

\section{Control and prediction of the organic solid state}

\section{“(2.3m, Price, University College London)}

This proposal seeks to understand the mysterious phenomenon of organic polymorphism, by developing a range of experimental and computational techniques to provide a complete atomistic description of the polymorphs of several organic molecules. These include the use of automated crystallisation techniques to find all likely polymorphs, diffraction technology, nuclear magnetic resonance, and computer simulation to predict possible structures and their physical properties".

Most researchers consider that the importance of these individual projects is their interdisciplinary nature and the desire of all the UKs Research councils to combine in such a programme that is aimed at embracing such a range of basic technologies. The projects chosen undoubtedly exhibit all the features of what may herald futuristic research, not only in the UK, but worldwide.

Professor B.H. Rudall Norbert Wiener Institute and University of Wales (UK) Section Editor 